\title{
On the Spherical Symmetry of the Electrical Conductivity of the Earth's Mantle
}

\author{
H. F. Petersons ${ }^{1}$ and R. S. ANDERSSEN ${ }^{2}$ \\ 'Department of Mathematics, Faculty of Science, The Australian National University, \\ Canberra A.C.T., Australia \\ ${ }^{2}$ Division of Mathematics and Statistics, Institute for Information Science and Engineering, \\ Commonwealth Scientific and Industrial Research Organization, and Centre for Mathematical Analysis, \\ Australian National University, Canberra A.C.T., Australia
}

(Received April 5, 1990; Revised August 1, 1990)

\begin{abstract}
Using the SCHMUCKeR (1970) methodology, an analysis of the magnitudes and phases of the $P_{1}^{0}$-response, for periods from 5 to 35 days, for a group of American and European observatories has been used to investigate the validity of the often assumed spherical symmetry of electrical conductivity of the Earth. The values of $\sigma$ and $z$, of the perfect substitute conductor determined by each observatory, were not found to be consistent with such a conductivity model thus supporting the suggestion of ROBERTS (1984) and indicating that a more complex model is required. Anomalous results were obtained for the North American high latitude region for which the test of SCHULTZ and LARSEN (1987) found response functions inconsistent with the assumption of a zonal morphology for the geomagnetic field, but our analysis discloses a geographical dependence of $\sigma$ and $z$ which may warrant further investigation. In the European region, the variation of $z$ suggests a general increase in the depth of the conducting mantle with decrease in geomagnetic latitude. The conductivity anomalies obtained from short period studies are not observed in our long period study. This is not unexpected as PRICE (1967) has shown that the surface anomalies are generally more transparent to the long period geomagnetic variations than the short period variations.
\end{abstract}

\section{Introduction}

In most studies of long period geomagnetic variations, the Earth is assumed to have a radially symmetric electrical conductivity structure (e.g. LAHIRI and PRICE, 1939; MCDONALD, 1957; BANKS, 1969, 1972; JADY, 1974; ANDERSSEN et al., 1979a, b; ACHACHE et al., 1981; JADY and PATERSON, 1983; JADY et al., 1983). The many regional analyses of short period geomagnetic variations (see PARKINSON (1983) for a review) see the presence of the oceans and the existence of regional conductivity anomalies in the layer which constitutes the upper $\sim 600 \mathrm{~km}$ of the mantle. In order to minimize such effects, we limit attention to long period geomagnetic variations.

ROBERTS (1984) used fourteen years of daily, three component geomagnetic data, recorded by 18 permanent geomagnetic observatories to estimate the response in the period range 2-200 days. The responses are found to be consistently and significantly different at different geographic locations and it is suggested that this is primarily due to the highly conducting oceans. The observation is also made however that stations geographically close to each other (and in roughly the same position with respect to the oceans) generally have similar response functions (e.g. WNG and FUR) but others (e.g. 
FUR and NGK) have estimated responses which are significantly and consistently different. It is argued that this is either due to the oceans or indicates that changes in the continental conductivity structure over fairly small distances can significantly affect the electromagnetic response even at long periods.

SCHULTZ and LARSEN (1987) used daily mean values spanning the period 1883-1980 and found response functions consistent with both the $P_{1}^{0}$ assumption and local 1-D Earth structure at only 22 of the 79 observatories examined. They do not do a spherical harmonic analysis study of the data but test the data using the assumption that if the source field is entirely zonal there would exist no time variations in declination. If significant variations in $D$ are found then the data are assumed to be inconsistent with zonal morphology. The problem is then to decide which variations are significant and which variations are due to greater intrinsic variability in the data as for example exists in data from high latitude observatories.

The present analysis is an attempt to investigate whether the response from the long period variations supports spherical symmetry at least as regards the sharp rise in electrical conductivity established for global conductivity models or whether the significantly different responses at neighbouring observatories found by ROBERTS (1984) are due to the changes in the continental conductivity structure. For this it is sufficient to examine the relative (rather than the actual) changes and justifies the use of the methodology of SCHMUCKER (1970) as outlined in the next section. This methodology has the advantage of simplicity and can thus serve to indicate changes in broad conductivity structures in a similar way that the CAGNIARD (1953) formulae may be used in magnetotelluric prospecting. It is realized that this approach is not always applicable and a more complex model may be needed to take into account the actual distribution of the inducing field and possible local complex conductivity structure. However, for this nonunique problem, given the presently available data, a more complex model does not seem to be justified.

In order to obtain estimates of the conductivity by this means, we need a sufficiently high density of observatory coverage. Two areas which have a high density coverage are Europe and North America. The European region has been found by ScHULTZ and LARSEN (1987) to have response functions consistent with both the $P_{1}^{0}$ assumption and local 1-D Earth structure. The North American region considered here does not satisfy their test criteria for zonal morphology but as there are a number of reasons why this test may lead to a negative result and the density of observatories in this region is high we investigate this region also.

\section{The Data and It's Analysis}

The data used were hourly values of $H$ and $Z$ at the geomagnetic observatories listed in Table 1 for the two year period 1964-1965 (see GUSTAFSON, 1984). For these observatories, the magnitude and phase of the $P_{1}^{0}$-response was calculated for periods of $5.01,10.04,15.06,20.08,24.98,30.12$ and 34.13 days. These periods were selected as they were long enough to be less likely to be influenced significantly by the oceanic effects and the very regional surface conductivity structures. Longer periods than the 34.13 days were not used as the two year data length used is likely to render the response estimates unreliable beyond that length. The more significant features of our analysis are that

a) hourly values and not daily means are used, 
Table 1. Observatories whose hourly values for $1964 / 65$ were used to compute the magnitude and phase of the $P_{1}^{0}$-response for select periods.

\begin{tabular}{|c|c|c|c|}
\hline Observatory & $\begin{array}{l}\text { Geomagnetic } \\
\text { latitude } \\
\text { (degrees) }\end{array}$ & Observatory & $\begin{array}{c}\text { Geomagnetic } \\
\text { latitude } \\
\text { (degrees) }\end{array}$ \\
\hline ALE (Alert) & 82.50 & NGK (Niemegk) & 52.07 \\
\hline HIS (Heiss Island) & 80.62 & VAL (Valentia) & 51.93 \\
\hline CCS (Cape Chelyuksin) & 77.72 & HAD (Hartland) & 51.00 \\
\hline THL (Thule) & 77.48 & KIV (Kiev) & 50.72 \\
\hline MBC (Mould Bay) & 76.20 & DOU (Dourbes) & 50.10 \\
\hline RES (Resolute Bay) & 74.70 & LVV (Lvov) & 49.90 \\
\hline DIK (Dixon Island) & 73.55 & VIC (Victoria) & 48.50 \\
\hline TRO (Tromso) & 69.67 & WIK (Wien-Kobenzl) & 48.27 \\
\hline GDH (Godhaven) & 69.23 & FUR (Furstenfeldbruck) & 48.17 \\
\hline SOD (Sodankyla) & 67.37 & ODE (Odessa-Stepanovka) & 46.78 \\
\hline CMO (College) & 64.87 & MMB (Memambetsu) & 43.90 \\
\hline BLC (Baker Lake) & 64.38 & AGN (Agincourt) & 43.78 \\
\hline DOB (Dombas) & 62.07 & PAG (Panagjurischte) & 42.52 \\
\hline NUR (Nurmijarvi) & 60.52 & AQU (L’Aquila) & 42.38 \\
\hline LER (Lerwick) & 60.13 & TIF (Tbilisi) & 42.08 \\
\hline LNN (Leningrad) & 59.95 & TKT (Tashkent) & 41.42 \\
\hline LOV (Lovo) & 59.35 & TOL (Toledo) & 39.88 \\
\hline SIT (Sitka) & 57.07 & FRD (Fredericksburg) & 38.20 \\
\hline RSV (Rude Skov) & 55.85 & ASH (Ashkabad) & 37.95 \\
\hline KZN (Kazan) & 55.83 & ALM (Almeria) & 36.85 \\
\hline MOS (Moscow) & 55.48 & KAK (Kakioka) & 36.23 \\
\hline ESK (Eskdailemuir) & 55.32 & SSO (Simosato) & 33.57 \\
\hline MEA (Meanook) & 54.62 & DAL (Dallas) & 32.98 \\
\hline MNK (Minsk) & 54.10 & TUC (Tucson) & 32.25 \\
\hline WNG (Wingst) & 53.75 & KNY (Kanoya) & 31.42 \\
\hline WIT (Witteveen) & 52.82 & & \\
\hline
\end{tabular}

b) two quiet years are used thus minimizing the random effects of storms especially for high latitude observatories,

c) homogeneous data is used (i.e. data for the same years 1964-5),

d) a high (for long period studies) density of coverage of observatories.

The actual analysis of the data was performed along the lines outlined in ANDERSSEN et al. (1979a, b) and GUSTAFSON (1984). For each of the selected periods $T$, the corresponding $Z / H$ (response) $=a(T)-i b(T)$ was used to compute the perfect substitute conductor conductivity $\sigma$ and depth of the perfect substitute conductor $z$ which, from the SCHMUCKER (1970) methodology for plane conductors, is given by

$$
\sigma(T)=T\left(4.45 \times 10^{4} \times b^{2}(T)\right) \quad \text { and } \quad z(T)=3185.5 \times a(T) .
$$

The above $\sigma(T)$ and $a(T)$ refer to the two layered model in which the top layer is assumed to be poorly conducting. The assumption is made that the incident variation field penetrates through this layer with negligible attenuation for the frequencies considered here and that the depth of penetration in the underlying substratum is small in comparison to the incident variation field's spatial wavelength. Thus, $z(T)$ represents the 
width of the poorly conducting layer and $\sigma(T)$ the conductivity of the good conducting layer as deduced from the response of the geomagnetic variation of period $T$.

\section{The Methodology and Its Interpretation}

We use the SCHMUCKER (1970) methodology to investigate the extent to which $\sigma$ and $z$ depend on (a) the time $T$ and (b) the geographical position. For long period observations (for which the oceans and local anomalies are invisible), it is well-known that, globally, the Earth's conductivity consists of a low conducting outer layer (crust and upper mantle) on a highly conducting inner region (inner mantle). If this conductivity structure of the Earth was spherically symmetric, then the dependence of $\sigma$ and $z$ on $T$ would be similar at observatories around the Earth. Furthermore if the dominant effect is the sharp rise in the electrical conductivity in the upper to lower mantle region we expect an increase in $\sigma$ and $z$ with increasing $T$. Such a radial conductivity model for the Earth will be referred to as model A.

The aim of this paper is to use the $\sigma$ and $z$ values derived for the observatories and periods of the previous section to examine the extent to which the Earth's conductivity has a spherically symmetrical structure. It will be seen to be a reasonable assumption, only if the $\sigma$ and $z$ values exhibit strong independence of geographic location and period $T$. If a dependence is observed, then it will be necessary to use this information to infer qualitatively the extent to which the Earth's conductivity differs from spherical symmetry as well as examine the ramifications of such a conclusion.

\section{Time Dependence}

At a single observatory, the dependence of $\sigma$ and $z$ on period, when plotted on the $z \sigma$-plane, gives a curve which will be referred to as the $\sigma z$-signature or simply the signature for that observatory (see Figs. 1(a) to 1(h)). From the behaviour of such signatures we aim to make inferences about the nature of the actual conductivity of the Earth. Due to the large range in $\sigma$ values for the North American observatories the signatures are plotted on three different scales. For all the Figs. 1(a) to 1(h), the same depth scale is used (i.e. one unit of depth corresponds to $100 \mathrm{~km}$ ). One $\sigma$ unit corresponds to $2000 \mathrm{~S} / \mathrm{m}$ in Fig. 1(a), $100 \mathrm{~S} / \mathrm{m}$ in Fig. 1(b), $0.4 \mathrm{~S} / \mathrm{m}$ in Fig. 1(c) while for the European observatories $1 \mathrm{~S} / \mathrm{m}$ in Figs. 1(d) to $1(\mathrm{~g})$, and for the Japanese observatories $0.1 \mathrm{~S} / \mathrm{m}$ in Fig. 1(h).

The signatures for the North American and high latitude observatories are given in Figs. 1(a), 1(b), and 1(c). The signatures for THL, GDH, DIK, MBC, RES, BLC, MEA, CMO, SIT and TRO (not plotted) are irregular (i.e. they do not show the expected trend for model A) and correspond to observatories in the region found by SCHULTZ and LARSEN (1987) to not satisfy their test for zonal morphology. The signatures are regular at DAL, TUC, FRD and VIC, AGN corresponding to observatories in the region found by SCHULTZ and LARSEN (1987) to be compatible with the $P_{1}^{0}$ and 1-D assumption. Note the flattening out of the curves at larger periods. In particular, observe the low $z$ of approximately $400 \mathrm{~km}$ for the flat portions of the curves for VIC and AGN compared with the substantially higher $z$ of approximately $800 \mathrm{~km}$ for the lower latitude observatories TUC, DAL, FRD. Such a result at a group of observatories is indicative of departure from spherical symmetry for the conductivity. Similar evidence is also found 
for the European observatories which are plotted in the Figs. 1(d) to $1(\mathrm{~g})$.

The European region has been found by SCHULTZ and LARSEN (1987) to have response functions consistent with both the $P_{1}^{0}$ assumption and local 1-D structure for the Earth. Hence the results from the SCHMUCKER (1970) methodology may be accepted with significantly greater confidence than for the North American region.

For the European region, the observatories have been divided into three groups. Figure 1(d) gives those observatories which have continually increasing $z$ with increasing $\sigma$ (that is show model A type behaviour). Figure 1(e) gives those observatories which have this trend except for $T=5$ days, and Figure 1(f) gives those observatories which have a general trend of decreasing $z$ with increasing $\sigma$. The latter two sets thus show departure from model $\mathrm{A}$ and indicate a more complex conductivity structure is being detected even by the 5 to 35 day responses.

A number of features may be observed in Figs. 1(d), (e), (f), namely,

(a) there is an increase in $\sigma$ with $T$ for all observatories in 1(d), an increase in $\sigma$ with $T$ for all observatories in 1(e) for $T>5$ days and also for observatories in 1(f),

(b) all observatories show an eventual flattening of the signatures as $T$ increases,

(c) in Fig. 1(d) which has signatures consistent with model A, we see (1) a general trend for $\sigma$ to increase for those observatories with greater $z,(2)$ there is also a general trend for $\sigma$ and $z$ to increase with decreasing latitude of the observatory.

Result (a) shows that the response for many European observatories is more complex than that of model A. Since SCHULTZ and LARSEN (1987) find that the response at mid-latitude observatories is due to a zonal $P_{1}^{0}$ source field, this variation at European observatories must be due to lateral inhomogeneities in the continental conductivity structure as suggested by ROBERTS (1984). Result (a) also indicates that even where more complex models than model A apply there is evidence for a significant increase in conductivity at some level in the Earth's mantle.

Result (b) is consistent with the assumption that there is a sharp increase in conductivity at some level in the upper mantle. The departure from a flat signature is most pronounced in the 5 day response even in the case of observatories consistent with model A indicating that the 5 to 10 day variations are subjected significantly to conductivity variations in the upper mantle and only periods generally longer than 5 to 10 days show the sharp rise in conductivity in the upper mantle.

However, the consistent general trend that is result (c) clearly indicates departure from spherical symmetry as does the geographic trends shown in Fig. 3. We can expect errors to arise in the response due to lateral inhomogeneity at a single observatory but would expect such errors to be random over the wider European region. This is not the case for the observatories of Fig. 1(d) which show a consistent trend over the European region.

Figure $1(\mathrm{~g})$ is a selection of observatories the signatures (except for the 5 day response) of which can be grouped geographically. Our findings are that NGK and FUR have similar signatures which however are consistently different as reported by ROBERTS (1984). FUR has consistently higher $z$ than NGK, however a far greater difference exists between the set NGK, FUR, DOU the set LER, ESK and the set TKT, ASH, TIF.

\section{Geographical Dependence}

Two regions of the Earth have a sufficiently close geographic distribution of 


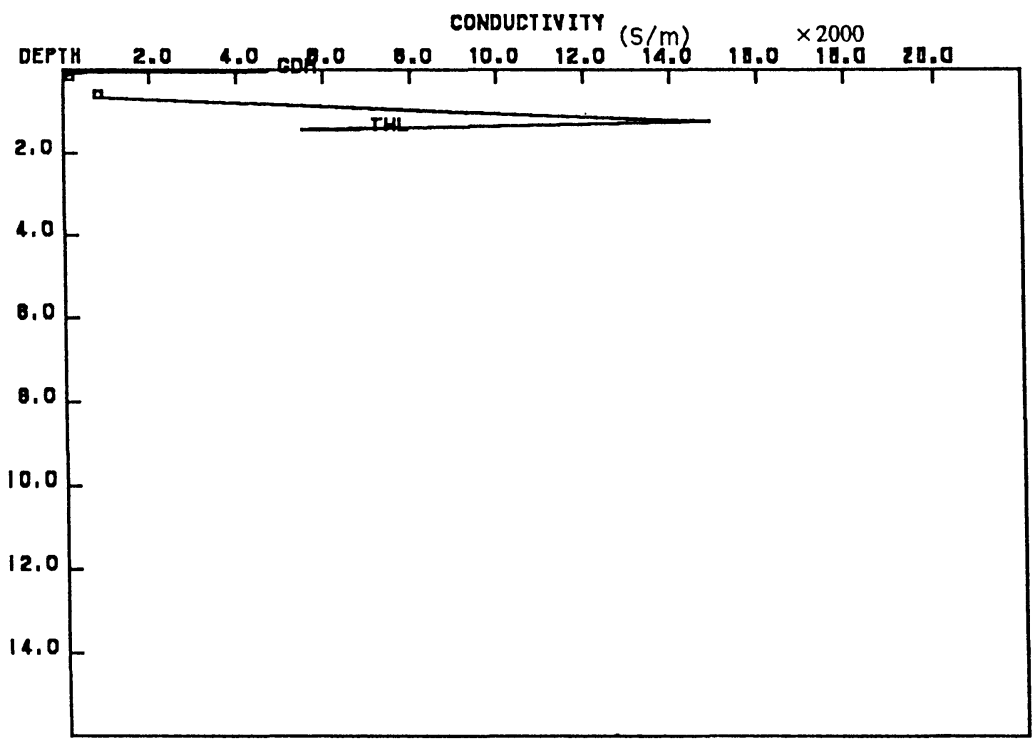

(km)

$\times 100$

(a)

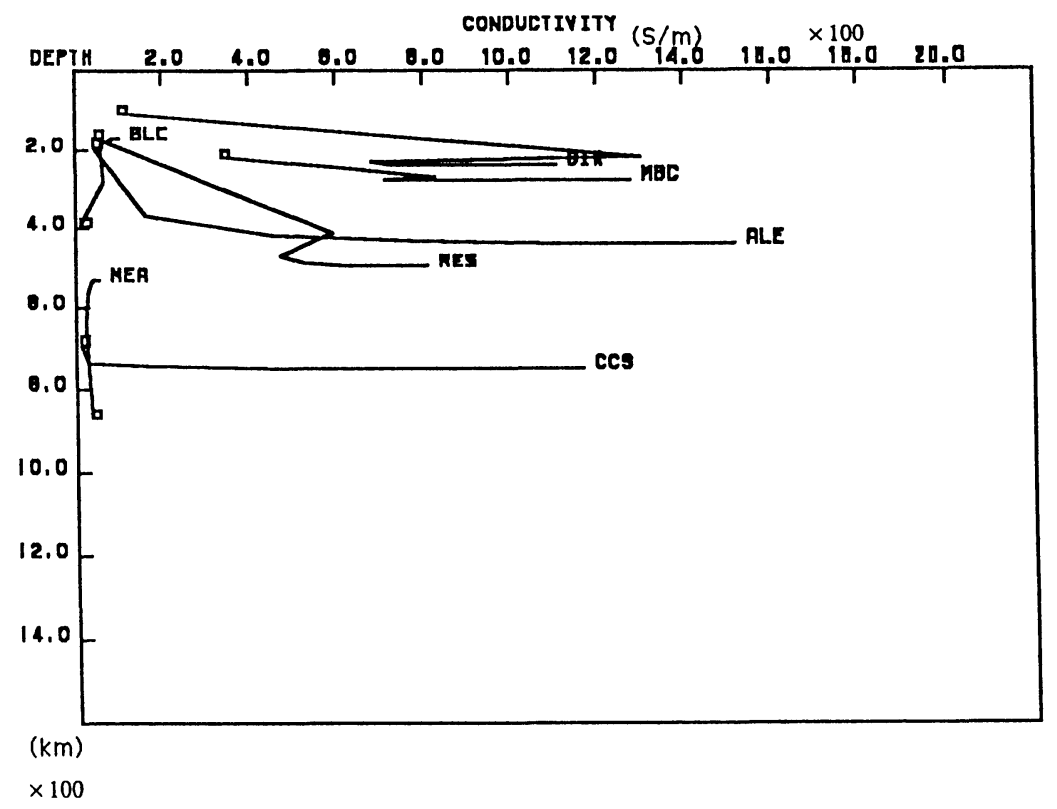

(b)

Fig. 1. Conductivity $\sigma$ versus depth $z$ signatures of the perfect substitute conductor for a number of European and North American observatories determined by geomagnetic variations with periods in the range 5 to 35 days. The starting value of 5.01 days is marked by a square. 


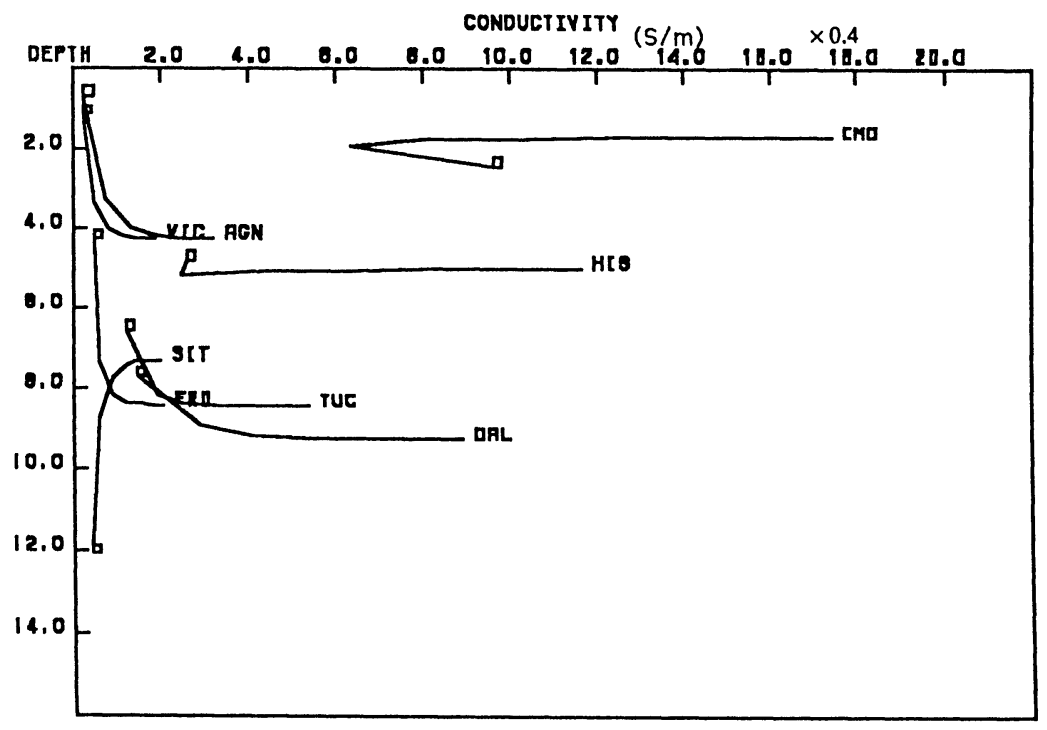

(km)

$\times 100$

(c)

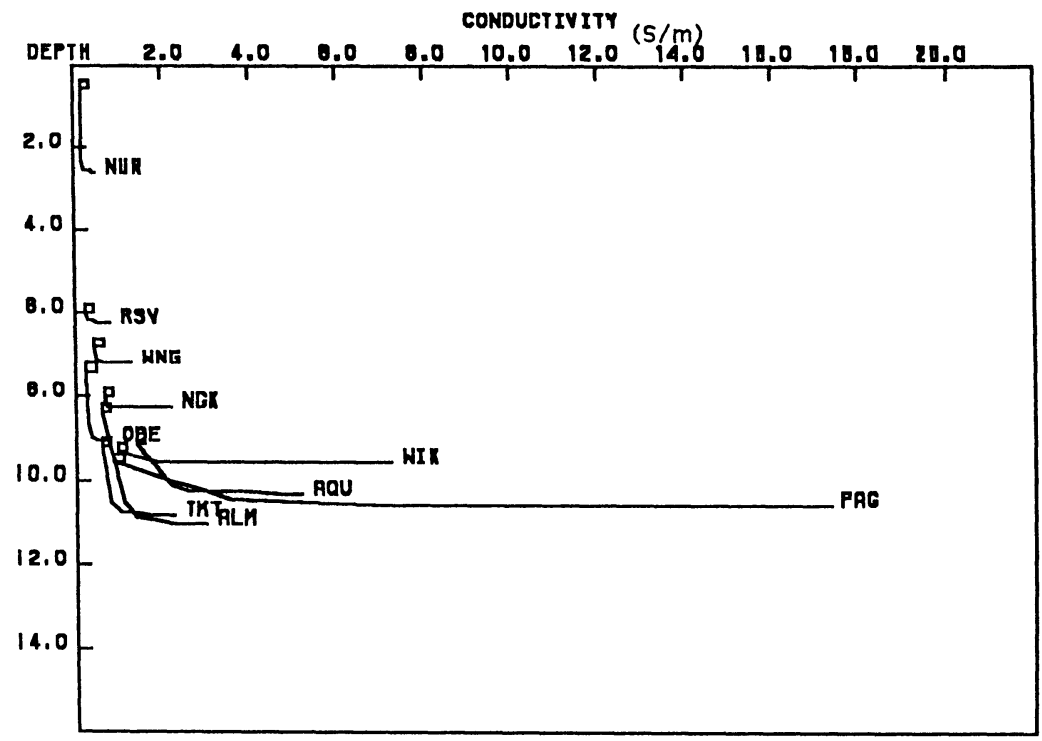

$(\mathrm{km})$

$\times 100$

(d)

Fig. 1. (continued). 


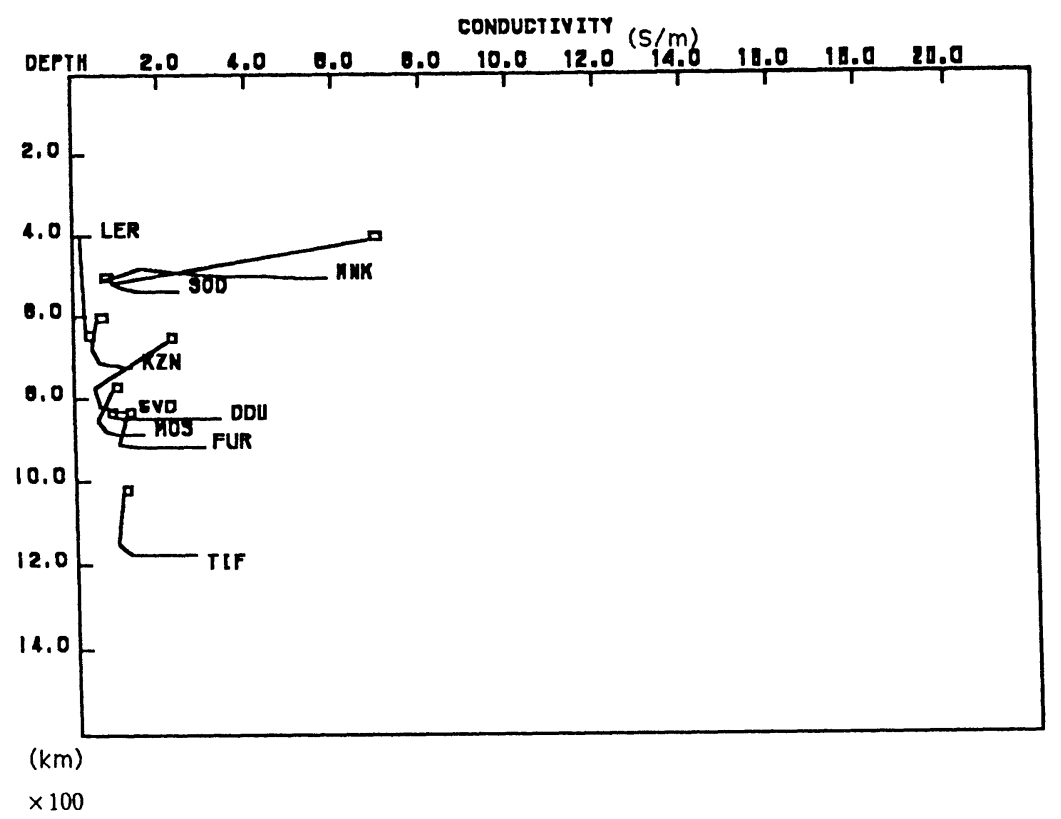

(e)

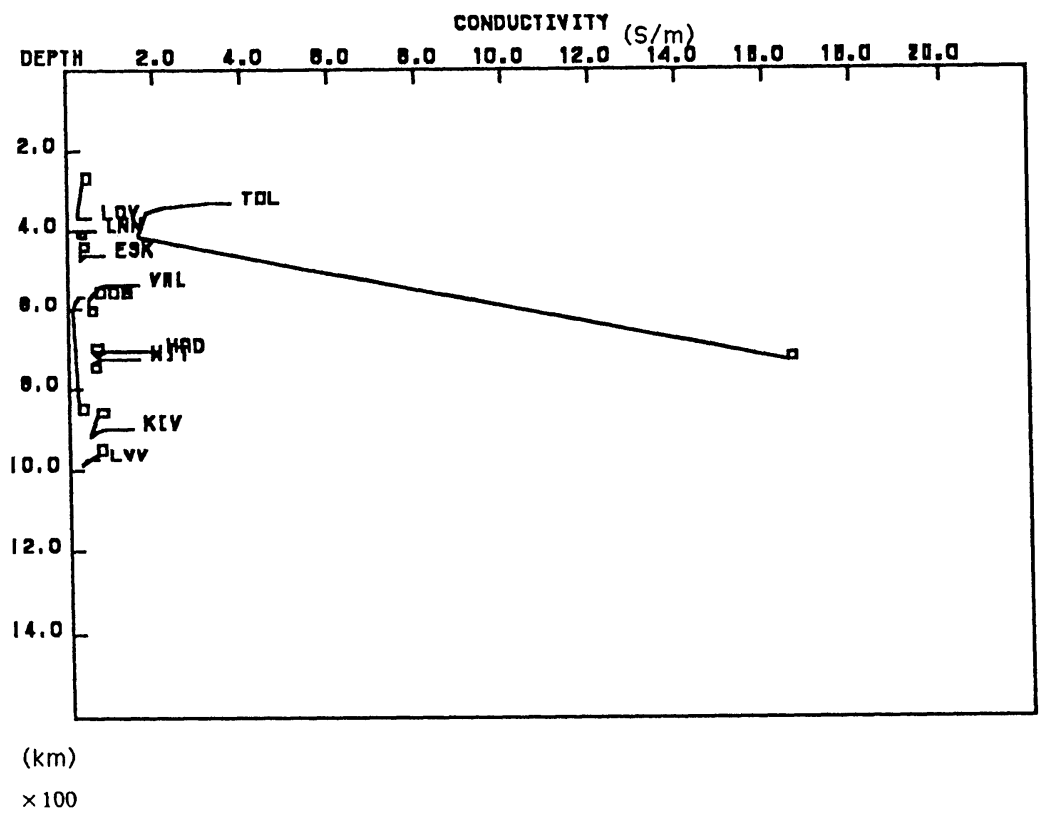

(f)

Fig. 1. (continued). 


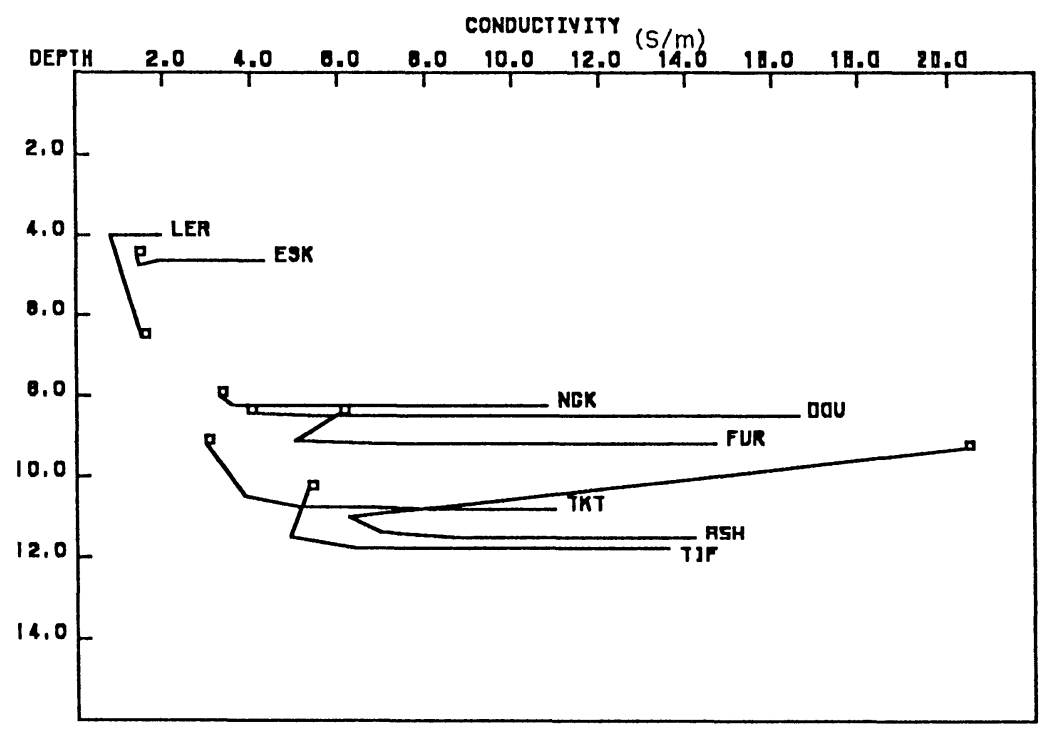

$(\mathrm{km})$

$\times 100$

(g)

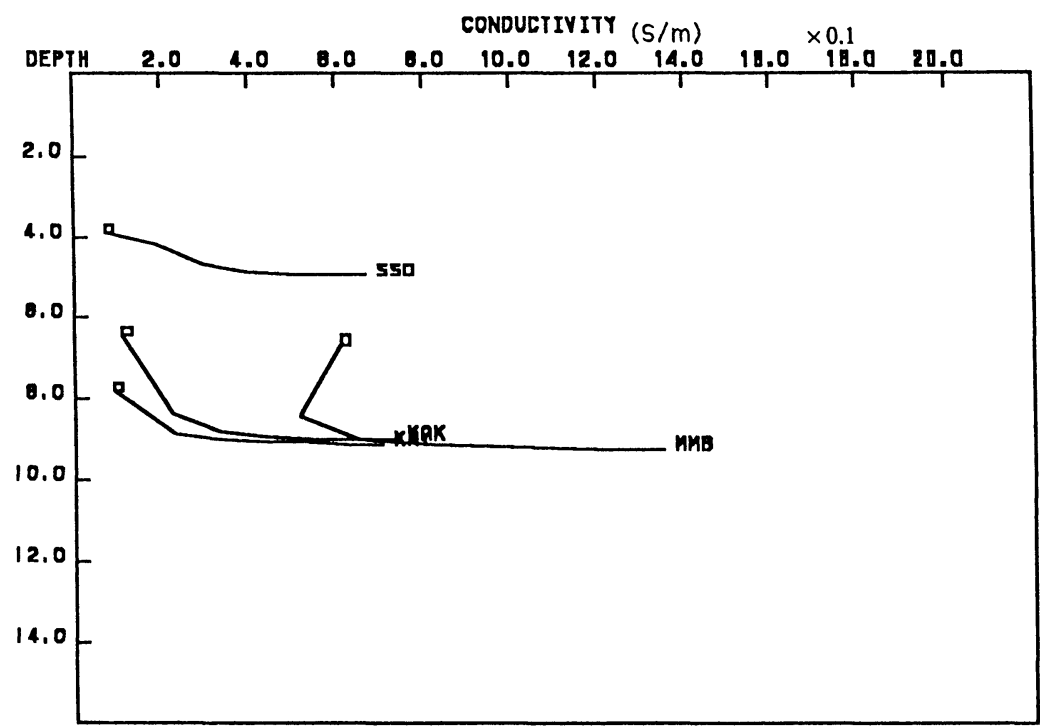

(km)

$\times 100$

(h)

Fig. 1. (continued). 
geomagnetic observatories to allow us to see if any general trend in both the geographic distribution of $\sigma$ and $z$ values exists. These are the North American region and the European region, which have some overlap. The North American observatories are plotted in Fig. 2 and the European in Fig. 3. A third region where some indication of the geographic distribution of $\sigma$ may be obtainable is the Japanese. Since the longer periods have the deeper penetration into the Earth, a plot was made (see Figs. 2 and 3) of the values of $\sigma$ and $z$ for the 34.13 day variation. The following may be observed.

For the North American region, we have a very highly conducting region near Thule-Godhaven where conductivity $\sigma$ of more than $9000 \mathrm{~S} / \mathrm{m}$ at depth $z \leq 150 \mathrm{~km}$ is observed. At Godhaven, we obtain the smallest value for $z$ of $6 \mathrm{~km}$. Both the conductivity and the depth values appear to be unrealistic since even for the lower mantle the conductivity is estimated as $200 \mathrm{~S} / \mathrm{m}$ (MCDONALD, 1957). One simple explanation is that these values are due to departure from zonal morphology as indicated by the test of SCHULTZ and LARSEN (1987). The departure from model A for this region, as indicated by Figs. 1(a), (b), (c), also tends to support this assumption. But the geographical dependence of $\sigma$ and $z$ is so regular in Fig. 2 that this is worth noting since it may have implications for the dependence of error on latitude if not for the conductivity of the Earth.

We then have a region of high conductivity indicated by the observatories RES, $\mathrm{MBC}$, ALE, CCS, DIK where $\sigma$ ranges from 122 to $228 \mathrm{~S} / \mathrm{m}$ and $z$ ranges from 270 to $750 \mathrm{~km}$. We have another region given by CMO, BLC, MEA, HIS, TRO where $\sigma$ ranges from 4.7 to $15 \mathrm{~S} / \mathrm{m}$ and where $z$ is low at TRO, CMO, and BLC ranging from 71 to 170 $\mathrm{km}$, but at HIS and MEA $z$ is $\sim 500 \mathrm{~km}$ indicating a tendency for low $z$ at some observatories but not at others in the same region. Outside this region the conductivity is lower than $4.7 \mathrm{~S} / \mathrm{m}$ and for the North American observatories $\sigma$ ranges from 0.7 to 3.5 $\mathrm{S} / \mathrm{m}$ and $z$ from 430 to $930 \mathrm{~km}$. Briefly, the general trend is a very high $\sigma$ at THL and GDH at shallow $z$, a decrease in $\sigma$ away from this region but with $z$ first showing an increase followed by possibly another decrease before increasing again.

When we turn to the European region we find generally low values of $\sigma$, the lowest $0.3 \mathrm{~S} / \mathrm{m}$ at DOB and highest at PAG of $17 \mathrm{~S} / \mathrm{m}$. The variation of $\sigma$ is found to be far from random as may be seen by the dashed lines of Fig. 3 which groups observatories with low, medium and high $\sigma$ values. Similarly for $z$, we see a regular trend in that there is general tendency for low, medium and high values to occur in certain regions. Thus, we may use $z$ to divide the European region into three regions as in Fig. 3, a low $z$ region where $z$ ranges from 71 to $540 \mathrm{~km}$, a medium $z$ region where $z$ ranges from 620 to $980 \mathrm{~km}$, and a high $z$ region where $z$ ranges from 1100 to $1200 \mathrm{~km}$.

For the Japanese region, only four observatories were used and Table 2 together with Fig. 1(h) summarizes the responses for SSO, KAK, KNY, MMB.

The range in $\sigma$ of 0.7 to $1.3 \mathrm{~S} / \mathrm{m}$ is small. The most noticeable effect is the low $z$ for SSO of $\sim 500 \mathrm{~km}$ compared to neighbouring observatories of $\sim 900 \mathrm{~km}$. Thus, our analysis using long period variations suggests that it is the change in depth of the high conductivity layer and not the conductivity which gives rise to the anomaly found by RIKITAKE (1966) using $D_{\text {st }}$ analysis but since only one station data namely SSO gives this effect further evidence is required if this result can be accepted with confidence. 


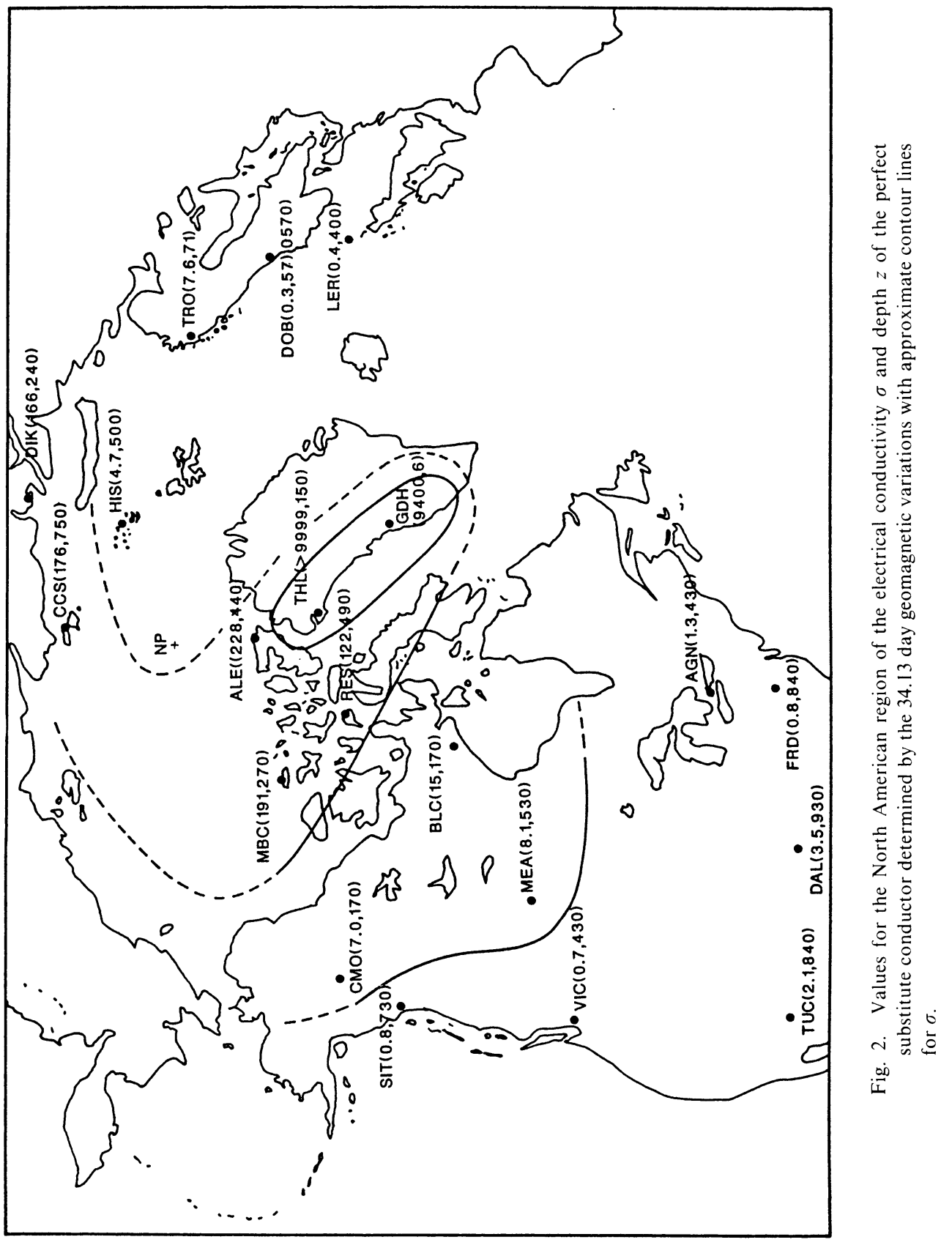



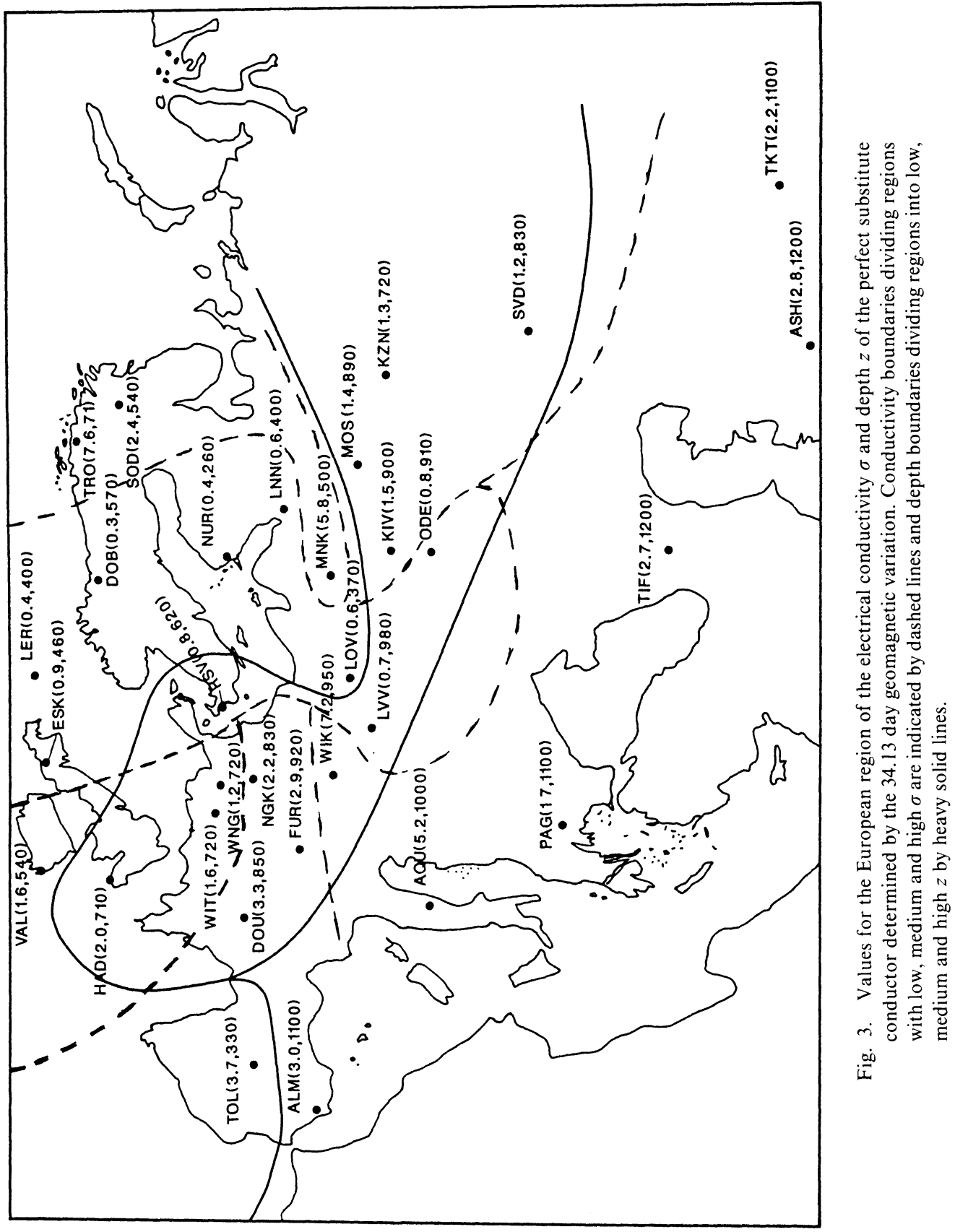
Table 2. Values of $\sigma$ and $z$ for four Japanese observatories.

\begin{tabular}{lcc}
\hline & $\sigma(\mathrm{S} / \mathrm{m})$ & $z(\mathrm{~km})$ \\
\hline MMB & 1.3 & 920 \\
KAK & 0.7 & 900 \\
KNY & 0.7 & 909 \\
SSO & 0.7 & 490 \\
\hline
\end{tabular}

\section{Data Length}

Even using hourly values, it appears that data sequences longer than two years are required to guarantee reasonably accurate and stable results. The importance of data length is shown in Table 3, where the analysis of one years data (of hourly values) at two European observatories is compared with the corresponding analysis for two years data. To a larger or lesser extent, the type of variability shown there is found to apply to all listed observatories of Table 1. In addition, when only one years data is used, the regular geographic dependence of $\sigma$ and $z$ of Figs. 2 and 3 is not observed for the North American high latitude observatories, and loses some of its clarity for the mid-latitude observatories of Europe and America.

These results confirm what is known theoretically. High data rates (e.g. hourly or shorter time steps) and long data lengths (e.g. two years or longer) are essential for ensuring accurate and stable results in the time series analysis of geomagnetic transient field data. Even using two years of hourly values, the results of the present analysis are not that accurate. Fortunately, however, there is sufficient accuracy to clearly identify the consistent geographic dependence of $\sigma$ and $z$ as outlined in this paper.

Table 3. $z(\mathrm{~km})$ and $\sigma(\mathrm{S} / \mathrm{m})$ values for $T=34.13$.

\begin{tabular}{|c|c|c|c|c|c|c|}
\hline Observatory & \multicolumn{2}{|c|}{1964} & \multicolumn{2}{|c|}{1965} & \multicolumn{2}{|c|}{$1964 \& 1965$} \\
\hline LER & 1150 & 0.2 & 450 & 0.1 & 400 & 0.4 \\
\hline ODE & 820 & 0.3 & 900 & 2.8 & 910 & 0.8 \\
\hline
\end{tabular}

\section{Comparison with Short Period Studies}

Numerous local conductivity anomalies using variations of a day or less have been made (see for example reviews by RIKITAKE (1971) and by PARKINSON (1983)). While it is not possible to rule out entirely the possibility that the global trends shown up in our analysis are not artifacts of regional anomalies the geographic consistency of these global trends makes such an outcome unlikely. Additional reasons for assuming that local conductivity anomalies are not the significant cause of the global conductivity anomalies is the behaviour of some of the local conductivity anomalies themselves.

Regional studies in the Canadian Arctic support our more global THL-GDH conductivity anomaly. Whitham and ANDERSEN (1965), NibleTt and Whitham (1970), pointed out that induction theory alone is incapable of explaining some of the 
observations on Ellesmere Island and suggest that induction over a larger area compared to the anomalous zones, with current distortion by local conducting bodies would be required to explain the observed local effects. DYCK and GARLAND (1969) studied this possibility by means of model experiments and showed that the main characteristics of the Alert anomaly may be explained in this way.

For the European region there also does not appear to be a correlation between short period conductivity anomalies and our long period results. For example, a short period anomaly has been observed at Eskdalemuir by OSEMEIKHIAN and EVERETT (1968) and HUTTON and JONES (1980). This is a linear anomaly which links the North Sea and Irish Sea across the Southern Uplands of Scotland. As may be seen from Fig. 3, no enhanced conductivity relative to other European observatories is found at Eskdalemuir for $T=34.13$, nor was it indicated for our other $T$ values all of which gave values for $\sigma$ of less than $0.9 \mathrm{~S} / \mathrm{m}$. A linear conductivity for northern Germany has been investigated by UNTIEDT (1970) which extends from near the mouth of the Rhine eastward and then south east into Poland. There is no indication of a near surface linear conductivity in Fig. 3, but there is a suggestion of a slight increase in $\sigma$ from north to south across Europe with a stronger dependence on latitude in $z$.

\section{Departure from Spherical Symmetry}

The present long period results show departure from simple spherical radial conductivity and suggest that a far more complex conductivity structure should be assumed for the Earth in two ways. The first is in the value of $z$, say $z^{\prime}$, at which high conductivity occurs as indicated by the flattening of the signatures in Figs. 1(a) to 1(h). Looking at the observatories in regions for which, as found by SCHULTZ and LARSEN (1987), both the $P_{1}^{0}$ assumption and local 1-D Earth structure applies we find that $z^{\prime}$ differs for different observatories in a nonrandom manner. This is true not only for individual observatories but for groups of observatories for the American region, Fig. 1(c), for the European region, Figs. 1(d) to 1(g), and Japanese region Fig. 1(h). For the European region especially, the $\sigma$ and $z$ values observed for the period $T=34.13$ days, Fig. 3, show a clearly nonrandom geographic variation for the depth and conductivity of the conducting mantle. The second is in the departures of the signatures from that of model A. In the case of the high latitude North American observatories this may be attributed to nonzonal morphology but for the European observatories where zonal $P_{1}^{0}$ morphology may be assumed this departure must be due to conductivity anomalies in the upper mantle as first suggested by ROBERTS (1984) which are different from the conductivity anomalies found from short period responses.

An offcentred conductosphere has been suggested by HERMANCE (1981) for the differences in the response functions at single observatories. However our analysis indicates a far more complex variation of $z^{\prime}$ than that of such an offcentred conductosphere.

Our results represent a possible explanation for the considerable variation in spherically symmetric conductivity models obtained by various workers (e.g. Fig. 112 in PARKINSON (1983)) in that the structure of such models will now depend on the geographical distribution of the observatories used. It is also natural to conclude from the above that a more detailed investigation of the type of methodology undertaken here is warranted especially for the anomalous high latitude North American observatories. 


\section{Conclusions}

Our analysis of variations with periods in the range 5 to 35 days using geomagnetic observatories indicates (a) that there is significant departure from a spherically symmetric conductivity distribution for the Earth, (b) a more complex variation of $\sigma$ with depth at many observatories than that of a relatively nonconducting layer overlying a conducting mantle whose conductivity increases with depth, (c) an extensive conductivity anomaly centred on Thule-Godhaven most likely due to departure from zonal morphology but requiring more detailed investigation for the reason for the observed regular geographic dependence, and (d) the importance of using at least two or more years of data.

We wish to thank Professor Sven-Ake Gustafson for carrying out the computations which form the basis of our analysis.

\section{REFERENCES}

Achache, J., J. L. LeMouel, and V. Courtillot, Long-period geomagnetic variation and mantle conductivity: An inversion using Bailey's method, Geophys. J. R. astr. Soc., 65, 579-601, 1981.

Anderssen, R. S., J. F. Devane, S.-A. Gustafson, and D. E. Winch, The qualitative character of the global electrical conductivity of the Earth, Phys. Earth Planet. Int., 20, 15-21, 1979a.

Anderssen, R. S., S.-A. Gustafson, and D. E. Winch, Estimating the phase of the response of the earth to long-period geomagnetic fluctuations, Earth Planet. Sci. Lett., 44, 1-6, 1979 b.

BANKS, R. J., Geomagnetic variations and the electrical conductivity of the upper mantle, Geophys. J. R. astr. Soc., 17, 457-487, 1969.

Banks, R. J., The overall conductivity distribution of the Earth, J. Geomag. Geoelectr., 24, 337-351, 1972.

CAgniard, L., Basic theory of the magnetotelluric method of geophysical prospecting, Geophysics, 18, $605-635,1953$.

Dyck, A. V. and G. D. Garland, A conductivity model for certain features of the Alert anomaly in geomagnetic variations, Can. J. Earth Sci., 6, 513-516, 1969.

GuSTAFSON, S.-A., On the determination of the electric conductivity of the Earth's interior from geomagnetic data, in Mathematical Programming and Numerical Analysis Workshop, edited by S.-A. Gustafson and R. S. Womersley, Proceedings of the Centre for Mathematical Analysis Aust. Nat. Uni., 6, pp. 89-101, 1984.

Hermance, J. F., Regionalization of global electromagnetic induction data: A theoretical model, Phys. Earth Planet. Inter., 27, 159-163, 1981.

Hutton, V. R. S. and A. G. Jones, Magnetovariational and magnetotelluric investigations in southern Scotland, J. Geomag. Geoelectr., 32, Suppl I, 141-150, 1980.

JADY, R. J., The conductivity of spherically symmetric layered Earth models determined by $S q$ and longer period magnetic variations, Geophys. J. R. astr. Soc., 36, 399-410, 1974.

JAdy, R. J. and G. A. Paterson, Inversion methods applied to Dst data, J. Geomag. Geoelectr., 35, 733-746, 1983.

JADY, R. J., G. A. PATERson, and K. A. Whaler, Inversion of the electromagnetic induction problem using Parker's algorithms with both precise and practical data, Geophys. J. R. astr. Soc., 75, 125-142, 1983.

LAhIRI, B. N. and A. T. Price, Electromagnetic induction in non-uniform conductors and the determination of the conductivity of the Earth from terrestrial magnetic variations, Phil. Trans. R. Soc. Lond. (A), 237, 509-540, 1939.

McDonald, K. L., Penetration of the geomagnetic secular field through a mantle with variable conductivity, $J$. Geophys. Res., 62, 117-141, 1957.

Niblett, E. R. and K. Whitham, Multi-disciplinary studies of geomagnetic variation anomalies in the Canadian Arctic, J. Geomag. Geoelectr., 22, 99-111, 1970.

Osemeik hian, J. E. A. and J. E. Everett, Anomalous magnetic variations in southern Scotland, Geophys. J. R. astr. Soc., 15, 361-366, 1968. 
Parkinson, W. D., Introduction to geomagnetism, 338 pp., Scottish Academic Press, London, 1983.

Price, A. T., Electromagnetic induction within the Earth, in Physics of Geomagnetic Phenomena, edited by S. Matsushita and W. H. Campbell, pp. 235-295, Academic Press, N.Y., 1967.

Rikitake, T., Electromagnetism and the Earth's Interior, 264 pp., Elsevier Publishing Co., London, 1966.

RIKITAKE, T., Electric conductivity anomaly in the Earth's crust and mantle, Earth Sci. Rev., 7, 35-65, 1971.

RoberTs, R. G., The long-period electromagnetic response of the Earth, Geophys. J. R. astr. Soc., 78, 547-572, 1984.

Schmucker, U., Anomalies of geomagnetic variations in the south-western United States, Bull. Scripps Inst. Oceanogr., 13, 165 pp., 1970.

Schultz, A. and J. C. LARSEN, On the electrical conductivity of the mid-mantle-1. Calculation of equivalent scalar magnetotelluric response functions, Geophys. J. R. astr. Soc., 88, 733-761, 1987.

UNTIEDT, J., Conductivity anomalies in central and southern Europe, J. Geomag. Geoelectr., 22, 131-149, 1970.

Whitham, K. and F. Andersen, Magnetotelluric experiments in northern Ellesmere Island, Geophys. J., 10, $317-345,1965$. 